\title{
Cálculo de la incertidumbre por simulación de Monte Carlo en la determinación de aflatoxina B1 en maní de exportación por HPLC-FD. Aplicación a la evaluación de la conformidad. Parte II
}

\author{
Gustavo Delgado ${ }^{1^{*}}$ y Norma Hernández ${ }^{2^{*}}$
}

1. Universidad Nacional Autónoma de Nicaragua, León (UNAN-LEÓN). Facultad de Ciencias y Tecnología, Departamento de Química, Laboratorio de Análisis de Trazas de Metales Pesados (LATMP), Edificio de Ciencias Básicas, León, Nicaragua. Tel: (505) 2311 5013, ext: 1132. Fax: (505) 23114012.

2. Laboratorio SER-AGRO S.A., Km 38 carretera Chinandega-Corinto, Tel: 23403493.

\section{RESUMEN}

El objetivo del presente estudio es utilizar la simulación numérica de Monte Carlo (SMC) para calcular la incertidumbre en la determinación de aflatoxina B1 (mensurando y) en una muestra de maní de exportación por Cromatografía Líquida de Alto Desempeño con Detector de Fluorescencia (HPLC-FD). El cálculo se basó en la generación de 5000 valores aleatorios para cada una de las 19 variables que influyen en el mensurando $y$, se introducen en la función que relaciona las variables y se obtienen 5000 valores aleatorios de la función. La incertidumbre se calculó como desviación estándar poblacional. El programa se elaboró en lenguaje Maple y en hoja de cálculo Excel, obteniéndose los mismos valores de incertidumbre, 0.225543 y 0.225180 . Estos resultados fueron comparados con los obtenidos por la aplicación de la ley de propagación de la incertidumbre y no hubo diferencias significativas. El contenido de aflatoxina B1 en la muestra de maní para exportación con su incertidumbre expandida fue $Y=(1.47 \pm 0.45) \mu \mathrm{g} / \mathrm{Kg}$ con un factor de cobertura de 2 al $95.45 \%$ de nivel de probabilidad. La evaluación de la conformidad del producto se basó en el enfoque funcional de la norma ISO 17000 y el requisito de los compradores, de acuerdo a la guía del cumplimiento con las especificaciones de la ILAC.

Palabras claves: incertidumbre por HPLC en maní, incertidumbre por simulación de Monte Carlo, evaluación de la conformidad.

\section{INTRODUCCIÓN}

En la primera parte de este estudio[1] se evaluó la incertidumbre en la determinación de aflatoxina B1 en una muestra de maní de exportación por Cromatografía Líquida de Alto Desempeño con Detector de Flourescencia (HPLC-FD), aplicando la ley de propagación de la incertidumbre ${ }^{[2-6]}$. La técnica de Monte Carlo es una alternativa para estimar la incertidumbre sin necesidad de realizar cálculos de derivadas parciales, tal a como lo establece el nuevo suplemento de la guía GUM ISO 2008 ${ }^{[7]}$.

Esta técnica es un método de simulación numérica (SMC) que sirve para generar variables aleatorias y evaluar la incertidumbre en sistemas complejos en diferentes campos de las ciencias ${ }^{[8]}$. Así por ejemplo, en la físico química teórica, en la mecánica cuántica y la mecánica estadística, donde muchos fenómenos están basados en modelos matemáticos difícilmente derivables, la técnica SMC es ampliamente utilizada ${ }^{[9]}$. En los últimos años la simulación de Monte Carlo ha venido aplicándose a la evaluación de la incertidumbre en el campo de la química analítica ${ }^{[8,10,11,23]}$.

Los requisitos de la norma para la competencia técnica de los laboratorios ISO/IEC 17025:2005 ${ }^{[12]}$ establece que los laboratorios de ensayos deben evaluar la incertidumbre en las mediciones, aplicando un procedimiento documentado. Los laboratorios deben presentar a sus clientes un informe de los resultados con su incertidumbre. Muchos clientes, sobre todo los compradores de productos de intercambio comercial y algunas instancias públicas, se preocupan por conocer la incertidumbre de las mediciones ${ }^{[13]}$ con el fin de evaluar la conformidad ${ }^{[14]}$ o cumplimiento con las especificaciones de un producto que ha sido evaluado utilizando el método seleccionado para el uso previsto $^{[14,15]}$ y basándose en los lineamientos de la guía para la evaluación y reporte del cumplimiento con la especificación ${ }^{[16]}$.

Los compradores de maní de exportación establecen sus requisitos para el contenido de aflatoxinas B1. Compradores ingleses establecen como criterio un contenido menor o igual a $1.5 \mu \mathrm{g} / \mathrm{Kg}$. En los Estados Unidos, al igual que la OMS, aceptan hasta $15 \mu \mathrm{g} /$ $\mathrm{Kg}$ como aflatoxinas totales (B1, B2, G1, G2) $)^{[1,17]}$. En el estudio que se realizó anteriormente ${ }^{[1]}$ se calculó la incertidumbre en la medición de aflatoxina B1 en la muestra de maní de exportación, siguiendo las guías GUM ISO 1995 y EURACHEM/CITAC ${ }^{[2,5]}$. Estas directrices establecen la ley de propagación de la incertidumbre ${ }^{[3,4]}$ como un método analítico para la 
evaluar la incertidumbre y son las que usualmente implementan los laboratorios de ensayos para calcular la incertidumbre de las mediciones. La norma ISO/IEC 17025 exige como requisito evaluar la incertidumbre y no especifica el procedimiento a utilizar; es el laboratorio el que debe establecer el procedimiento normalizado para evaluar la incertidumbre.

Por lo tanto, en el presente trabajo se pretende proponer la técnica de Simulación de Monte Carlo como una alternativa para evaluar la incertidumbre en la determinación de aflatoxina B1 en maní de exportación por HPLC-FD y aplicarla a la evaluación de la conformidad del producto de acuerdo con los requisitos de los compradores. Para ello, se tomaron los resultados del trabajo anterior ${ }^{[1]}$, se elaboró un algoritmo para el cálculo de la incertidumbre por SMC, se programó en lenguaje Maple ${ }^{[18]}$ y en hoja de cálculo Excel, y se compararon los resultados con las especificaciones establecidas por el cliente.

\section{TEORÍA}

Ya se ha discutido ${ }^{[1]}$ que existen dos vías para evaluar la incertidumbre en las mediciones: por métodos analíticos aplicando la ley de la propagación de la incertidumbre y por métodos numéricos utilizando la técnica de simulación de Monte Carlo $^{[7]}$ que propaga las distribuciones de las diferentes variables de entrada. En lo que sigue se describe brevemente cada una de estas técnicas. En la figura 1 se ilustra la ley de la propagación de la incertidumbre para 3 variables de entrada. Cada variable de entrada $x_{i}$ es caracterizada por su esperanza y su incertidumbre asociada $u_{x i}$ siguiendo una función de densidad de probabilidad. Esta información se propaga utilizando la ley de propagación de la incertidumbre a través del desarrollo de la expansión de Taylor, despreciando las derivadas mayores de segundo orden ${ }^{[2]}$.

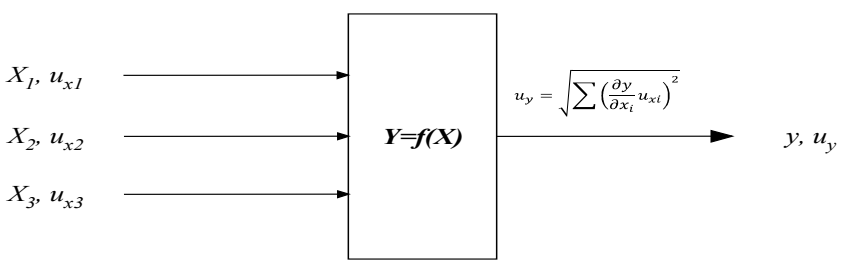

Figura 1. Ilustración de la ley de propagación de la incertidumbre de tres variables independientes.

En la figura 2 se ilustra la propagación de las distribuciones de tres variables independientes. Cada variable de entrada está caracterizada por una función de densidad de probabilidad PDF $g_{x i}(\xi)$, con distribución normal según la ecuación siguiente:

$$
g_{x}(\xi)=\frac{1}{\sigma \sqrt{2 \pi}} \exp \left[-\frac{1}{2}\left(\frac{\xi-\mu}{\sigma}\right)\right]
$$

Las tres PDFs $g_{x i}\left(\xi_{i}\right), i=1,2,3$, se propagan a través de la simulación de Montecarlo para obtener un mensurando $y$ con distribución gaussiana y una función de densidad de probabilidad $g_{y}(\eta)$.



Figura 2. Ilustración de la propagación de las distribuciones (por la técnica Monte Carlo) para tres variables independientes.

Pueden existir diferentes distribuciones de entradas (gaussiana, rectangular, $t$, etc.) y la PDF del mensurando obtenida podría tener forma asimétrica ${ }^{[7]}$.

El proceso del cálculo de la incertidumbre, utilizando la simulación de Monte Carlo se puede representar en el esquema de la figura 3 , partiendo del modelo que relaciona el mensurando con la función de las variables y finalizando con el valor del mensurando $y$ y su incertidumbre expandida $U$ con un factor de cobertura $k(=2)$ al $95.45 \%$ de probabilidad de cobertura.

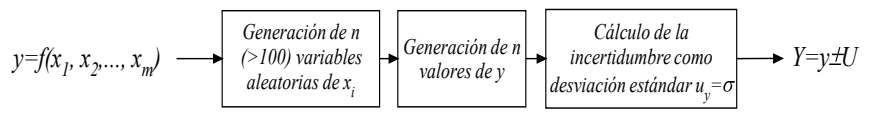

Figura 3. Proceso del cálculo de la incertidumbre por SMC.

Este proceso se puede resumir así: a partir de la generación de una población de variables aleatorias de entradas con distribución gaussiana, se genera una población normal del mensurando y se calcula su incertidumbre. El principio del cálculo se detalla a continuación.

\section{PRINCIPIO DE LA TÉCNICA DE SIMULACIÓN DE MONTE CARLO (SMC)}

La técnica de Monte Carlo está inspirada en los juegos de azar (poker), y se le ha denominado Monte Carlo por la capital de Mónaco, donde existen los famosos casinos del Principado ${ }^{[7,8]}$. Esta técnica se resume en las siguientes etapas ${ }^{[7]}$ :

\section{Formulación:}

a)Se define el mensurando $Y$ o la variable de salida.

b)Se determinan las variables de entrada $X=x_{1}, x_{2}, \ldots, x_{m}$ que influyen en el mensurando.

c) Se deduce el modelo que relaciona $Y$ con $X, y=f\left(x_{j}\right)$. Siendo $j=1,2, \ldots, m$ variables.

d)De acuerdo al tipo de distribución (normal, rectangular, etc.) se le asignan los PDFs a las variables $x_{j}$. Normal para el presente estudio.

Universitas 3 [1] 2009, ISSN 2071-2574 | 17 


\section{Propagación de las funciones de densidad de probabilidad (PDFs):}

Para $x_{j}$ a través del modelo $f\left(x_{j}\right)$, para obtener la PDF de $Y$.

a)Para cada variable $x$ se genera una variable aleatoria de distribución normal a partir de un número aleatorio de distribución uniforme: $v_{i j}=x_{i}+z_{i j} u_{j}$ (ver anexo).

b)Se generan $n$ valores aleatorios de la función $y_{i}=f\left(v_{i j}\right)$ caracterizado por una distribución de probabilidad gaussiana $N\left(y, u_{y}\right)$ :

$$
y=\left[\begin{array}{c}
f_{1}\left(v_{11}, v_{12}, \ldots, v_{1 m}\right) \\
f_{i}\left(v_{i j}\right) \\
f_{n}\left(v_{n 1}, v_{n 2}, \ldots, v_{n m}\right)
\end{array}\right]
$$

\section{Valores característicos del mensurando:}

a)Se obtiene el estimado o esperanza de $Y$.

b)Se obtiene la desviación estándar de $Y$, se toma como la incertidumbre estándar $u_{y}$ asociada con $y$.

c)Se obtiene el intervalo de cobertura que contiene a $Y$ con una probabilidad especificada (probabilidad de cobertura). En el presente estudio asumimos un factor de cobertura $k=2$, admitiendo una PDF gaussiana.

\section{EVALUACIÓN DE LA CONFORMIDAD (EC)}

Uno de los principales obstáculos técnicos al comercio internacional son las normas técnicas que establecen los requisitos para la adquisición de un producto o de un servicio. Para evitar estos obstáculos la Asociación Latinoamericana de Integración (ALADI) ha suscrito un "acuerdo de superación a los obstáculos técnicos al comercio"[21], con el fin de crear y fortalecer los sistemas de evaluación de la conformidad (SEC) para viabilizar el reconocimiento mutuo de los SEC, tomando como base recomendaciones de organismos internacionales especializados, tales como la ISO, el Foro Internacional de Acreditación (IAF) y la Cooperación Internacional de Acreditación de Laboratorios (ILAC). A la vez, se comprometen a fortalecer las estructuras de EC y a utilizar la capacidad de los laboratorios de la región.

El principio de la evaluación de la conformidad está basado en el enfoque funcional[14], caracterizado por una serie de funciones que satisfacen los requisitos especificados por el cliente: selección, determinación y revisión y atestación. Los compradores establecen sus requisitos para la adquisición del producto[1]; el laboratorio debe seleccionar el método apropiado, proceder a la determinación y revisar los resultados para proporcionar una constancia o atestación de la conformidad. Esto significa que el laboratorio evaluador de la conformidad debe garantizar que los resultados de sus ensayos sean trazables, precisos y confiables. El producto va acompañado con la constancia del laboratorio como evidencia del cumplimiento de la conformidad $\mathrm{o}$ especificaciones ${ }^{[16]}$ requeridas por el cliente.

La incertidumbre expandida es un factor muy importante en el cumplimiento de la conformidad. El nivel de incertidumbre aceptable tiene que decidirse sobre la base de la aptitud para su uso, y esta decisión se toma a partir de un acuerdo con el cliente ${ }^{[13]}$. La guía para la evaluación y reporte del cumplimiento con las especificaciones ${ }^{[16]}$, presenta los diferentes casos que establecen los criterios (basados en la incertidumbre expandida al $95 \%$ de nivel de confianza) para la toma de decisiones en el cumplimiento con la conformidad del producto.

La tabla I muestra los diferentes casos con los resultados de los ensayos y su incertidumbre expandida frente a los límites superior o inferior de la especificación (LES y LEI) del producto y el cumplimiento con los requisitos del cliente.

Tabla I. Criterios de cumplimiento de los resultados de ensayos con las especificaciones del producto y los requisitos del cliente. Cumplimiento posible SI o posible NO, indica decisión por acuerdo con el cliente.

\begin{tabular}{|c|c|c|c|}
\hline Caso & $\begin{array}{c}\text { Resultados al } 95 \% \\
\text { de probabilidad }\end{array}$ & Requisitos* & Cumplimiento \\
\hline 1 & $y+U<L E S$ & $\begin{array}{l}<L E S \\
\leq L E S\end{array}$ & $\begin{array}{l}\mathrm{Si} \\
\mathrm{Si}\end{array}$ \\
\hline 2 & $y<L E S<y+U$ & $\begin{array}{l}<L E S \\
\leq L E S\end{array}$ & $\begin{array}{l}\text { Posible No } \\
\text { Posible Si }\end{array}$ \\
\hline 3 & $y=L E S$ & $\begin{array}{l}<L E S \\
\leq L E S\end{array}$ & $\begin{array}{l}\text { Posible No } \\
\text { Posible Si }\end{array}$ \\
\hline 4 & $y>L E S>y-U$ & $\begin{array}{l}<L E S \\
\leq L E S\end{array}$ & $\begin{array}{l}\text { Posible No } \\
\text { Posible No }\end{array}$ \\
\hline 5 & $y-U>L E S$ & $\begin{array}{l}<L E S \\
\leq L E S\end{array}$ & $\begin{array}{l}\text { No } \\
\text { No }\end{array}$ \\
\hline 6 & $y-U>L E I$ & $\begin{array}{l}<L E I \\
\leq L E I\end{array}$ & $\begin{array}{l}\mathrm{Si} \\
\mathrm{Si}\end{array}$ \\
\hline 7 & $y>L E I>y-U$ & $\begin{array}{l}<L E I \\
\leq L E I\end{array}$ & $\begin{array}{l}\text { Posible No } \\
\text { Posible Si }\end{array}$ \\
\hline 8 & $y=L E I$ & $\begin{array}{l}<L E I \\
\leq L E I\end{array}$ & $\begin{array}{l}\text { Posible No } \\
\text { Posible Si }\end{array}$ \\
\hline 9 & $y<L E I<y+U$ & $\begin{array}{l}<L E I \\
\leq L E I\end{array}$ & $\begin{array}{l}\text { Posible No } \\
\text { Posible No }\end{array}$ \\
\hline 10 & $y+U<L E I$ & $\begin{array}{l}<L E I \\
\leq L E I\end{array}$ & $\begin{array}{l}\text { No } \\
\text { No }\end{array}$ \\
\hline
\end{tabular}

*Especificaciones del cliente 


\section{METODOLOGÍA}

Todas las mediciones fueron efectuadas con los instrumentos calibrados con patrones de trabajo trazables al sistema SI y con materiales de referencia certificados. El detalle del procedimiento y las condiciones de operación del HPLC se encuentran en el estudio anterior ${ }^{[1]} y$ en los documentos del laboratorio ${ }^{[22]}$.

El equipo utilizado fue un cromatógrafo HPLC HP1050 con detector de flourescencia (FD) HP-1046A y con un reactor quimioluminiscente post columna. Los materiales, reactivos y solventes están detallado en el procedimiento del laborotorio ${ }^{[22]}$. Las soluciones patrones se prepararon a partir de soluciones de referencias certificadas. La balanza (con resolución $\pm 0.01 \mathrm{~g}$ ) y los volumétricos utilizados estaban calibrados, con su certificado de calibración trazable al SI. La variación de temperatura ambiental se registró con un termómetro calibrado trazable al SI.

El procedimiento analítico consistió en preparar una disolución de la muestra de maní con metanol-agua, se extrajo la aflatoxina B1 con cloroformo, se evaporó y se redisolvió en metanol-agua, y por último se efectuó el proceso cromatográfico para medir el área de pico.

Por otro lado, se obtienen los cromatogramas del blanco y de la solución de referencia a un nivel de concentración de $5 \mu \mathrm{g} / \mathrm{L}$. Con las áreas de picos y los variables experimentales se realizó el cálculo del mensurando con su incertidumbre asociada mediante el modelo matemático apropiado[1].

\section{RESULTADOS Y DISCUSIÓN}

\section{Procedimiento modificado para el cálculo de la incertidumbre}

El procedimiento para el cálculo de la incertidumbre en la determinación de aflatoxina B1 siguiendo la guía GUM ISO 1995 fue ampliamente discutido en el estudio anterior ${ }^{[1]}$. En el presente trabajo se propone modificar el procedimiento, sustituyendo el proceso que se basa en la ley de propagación de la incertidumbre por el de la simulación de Monte Carlo. El diagrama de flujo de este procedimiento se presenta la figura 4.

Se define el mensurando y se identifican las variables que influyen, se deduce el modelo matemático, se identifican las fuentes de incertidumbres a través del diagrama causa-efecto, se cuantifican las incertidumbres de tipo A y/o B, se calcula la incertidumbre del mensurando $u_{v}$ por la técnica SMC, se revisa si no hay incongruencias para reevaluar los componentes de la incertidumbre, se calcula la incertidumbre expandida con el factor de cobertura $k=2$ al $95.45 \%$ de nivel de probabilidad y se reporta el intervalo de cobertura con el número de cifras significativas correcto.

\section{Aplicación del procedimiento}

La aplicación del procedimiento modificado se efectuará en base a los resultados del trabajo anterior ${ }^{[1]}$. El detalle de las operaciones del procedimiento se presenta a continuación.

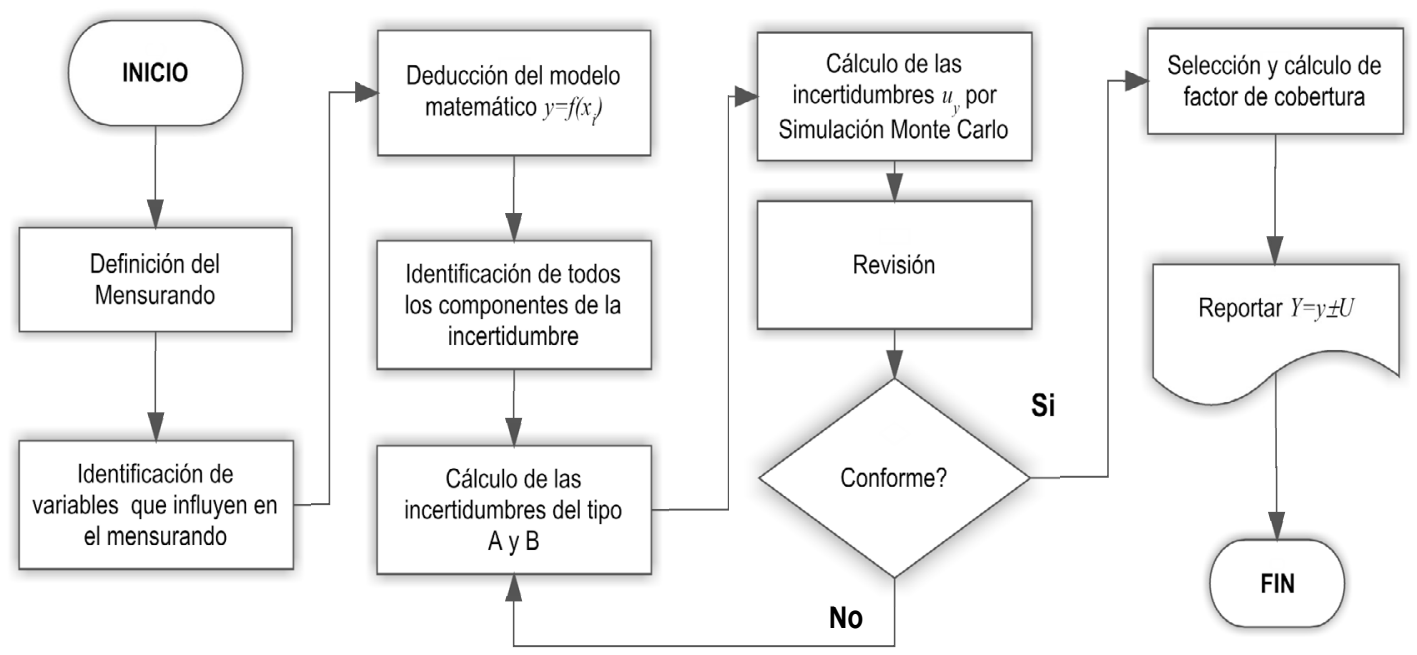

Figura 4. Diagrama de flujo del procedimiento para calcular la incertidumbre por simulación de Monte Carlo. 


\section{Definición del mensurando y variables que influyen}

El mensurando $y$ es el contenido de aflatoxina B1en $\mu \mathrm{g} /$ $\mathrm{Kg}$ en la muestra de maní de exportación. Las variables que influyen en el mensurando se presentan en la tabla II.

Tabla II: Variables que influyen en el mensurando $y$

\begin{tabular}{|c|c|}
\hline $\begin{array}{c}\text { Variable } \\
x_{i}\end{array}$ & Especificación (unidades) \\
\hline$A x$ & $\begin{array}{l}\text { Área de pico de aflatoxina B1 en la solución } \\
\text { muestra (LU-S) }\end{array}$ \\
\hline As & $\begin{array}{l}\text { Área de pico de aflatoxina B1 en la solución de } \\
\text { referencia (LU-S) }\end{array}$ \\
\hline$C_{M R C}$ & $\begin{array}{l}\text { Concentración de aflatoxina B1 de la solución de } \\
\text { referencia certificada ( } \mathrm{mg} / \mathrm{L})\end{array}$ \\
\hline$P$ & $\begin{array}{l}\text { Pureza de la aflatoxina B1 en la solución de } \\
\text { referencia }(P \% / 100)\end{array}$ \\
\hline$v_{01}$ & $\begin{array}{l}\text { Alícuota de la solución de referencia certificada } \\
(\mathrm{mL})\end{array}$ \\
\hline$V_{02}$ & $\begin{array}{l}\text { Volumen de dilución con metanol para la solución } \\
\text { de referencia }(m L)\end{array}$ \\
\hline$V_{03}$ & $\begin{array}{l}\text { Alícuota de la solución de referencia diluida en } \\
\text { v02 (mL) }\end{array}$ \\
\hline$V_{04}$ & Volumen de agua agregado a v03 (mL) \\
\hline$V_{05}$ & Volumen de metanol agregado a v04 (mL) \\
\hline$V_{s}$ & $\begin{array}{l}\text { Volumen de "slurry" (mezcla acuosa) de harina de } \\
\text { muestra de maní ( } m L \text { ) }\end{array}$ \\
\hline$V_{0}$ & Volumen metanol agregado a $\mathrm{Vs}(\mathrm{mL})$ \\
\hline$V_{1}$ & Volumen recolectado de la filtración de VO $(\mathrm{mL})$ \\
\hline$V_{2}$ & $\begin{array}{l}\text { Volumen de salmuera al } 20 \% \text { agregado a V1 } \\
(\mathrm{mL})\end{array}$ \\
\hline$V_{3}$ & $\begin{array}{l}\text { Volumen recolectado de la filtración de (V2+V1) } \\
(m L)\end{array}$ \\
\hline$V_{4}$ & Volumen de cloroformo agregado a V3 (mL) \\
\hline$V_{5}$ & $\begin{array}{l}\text { Volumen de cloroformo recolectado después de } \\
\text { separación }(m L)\end{array}$ \\
\hline$V_{6}$ & Volumen añadido de metanol-agua $(\mathrm{mL})$ \\
\hline$w$ & Peso de la muestra de harina de maní $(g)$ \\
\hline$R_{c}$ & Recuperación (\%) \\
\hline
\end{tabular}

\section{Modelo matemático}

El modelo matemático ha sido deducido a partir de las etapas del procedimiento analítico ${ }^{[1,24]}$.

$f=\left(\frac{A_{x}}{A_{s}}\right)\left(\frac{C_{M R C} P v_{01} v_{03}}{v_{02}\left(v_{03}+v_{04}+v_{05}\right)} \times 1000\right)\left(\frac{V_{4}\left(V_{1}+V_{2}\right)\left(V_{0}+V_{S}\right) V_{6}}{V_{5} V_{3} V_{1} w}\right)\left(\frac{100}{R c}\right)$

\section{Incertidumbres de las variables del modelo}

Las fuentes de incertidumbres ya se han identificado y calculado anteriormente ${ }^{[1]}$. El diagrama causaefecto fue aplicado para identificar las fuentes, y los valores respectivos han sido calculado de acuerdo al tipo de incertidumbre; las de tipo A se obtuvieron por la desviación estándar de una serie de observaciones y las de tipo $B$ se calcularon según la distribución (normal, rectangular o triangular). El valor de las variables y las incertidumbres se encuentran en la tabla III. La incertidumbre de la pureza $(P=99 / 100=0.99)$ de la solución de referencia no está especificada en el certificado. Por lo tanto se puede asumir una incertidumbre expandida unitaria en el último dígito con una distribución rectangular, es decir $u_{y}=0.01 / \sqrt{ } 3$.

Tabla III: Variables del mensurando y sus incertidumbres

\begin{tabular}{ccc}
\hline Variable $x_{i}$ & Valor & $u_{x i}$ \\
\hline$A x$ & 12.22328 & 1.47821886 \\
$A s$ & 76.09827 & 1.10384003 \\
$C_{M R C}$ & 3.415 & 0.01443376 \\
$v_{01}$ & 1 & 0.05720036 \\
$V_{03}$ & 1 & 0.05720036 \\
$V_{02}$ & 10 & 0.02328687 \\
$V_{04}$ & 25 & 0.01709702 \\
$V_{05}$ & 25 & 0.05640609 \\
$V_{4}$ & 3.5 & 0.14516939 \\
$V_{1}$ & 30 & 0.0539409 \\
$V_{2}$ & 20 & 0.02188784 \\
$V_{0}$ & 200 & 0.92813358 \\
$V_{s}$ & 100 & 0.0369414 \\
$V_{6}$ & 1.6 & 0.02093419 \\
$V_{5}$ & 1.6 & 0.00585692 \\
$V_{3}$ & 40 & 0.21637327 \\
$w$ & 33.33 & 0.00763763 \\
$R c$ & 94.78 & 0.050100 \\
$P_{*}$ & 0.99 & 0.0057735 \\
\hline
\end{tabular}

*incertidumbre no especificada

\section{Cálculo de la incertidumbre $u_{v}$ por simulación de Monte Carlo}

En la figura 3 se presentó el esquema general para el cálculo de la incertidumbre por simulación numérica de Monte Carlo. Se generan los valores de las variables aleatorias que influyen en el mensurando $y$, se introducen en la función $\mathrm{f}$ del modelo matemático (ecuación 1) y se calcula el valor de $y$. El proceso se repite 5000 veces y se calcula la incertidumbre como desviación estándar.

\section{Cálculo usando Maple}

El algoritmo para generar valores aleatorios del mensurando $y$ se puede ver en el diagrama de flujo de la figura 5. Primero se introducen los valores de 
Figura 5. Algoritmo de SMC para el cálculo de la incertidumbre del mensurando $u_{v}$ en lenguaje Maple.



las variables $x_{j}$ del modelo a través de la función $f$, $y=f\left(x_{i}\right)$ donde $x_{j}$ son las variables experimentales que se encuentran en la tabla III, $j=1,2 \ldots, m$, siendo $m=19$ variables que tiene la función $f$ (ecuación 1). Se asigna $\mathrm{n}=5000$ para generar poblaciones de valores aleatorias $v_{i j}$ a partir de los valores de las variables $x_{i}$, utilizando el algoritmo $v_{i j}=x_{j}+z_{i j} u_{j}$; los valores de la variable $x_{j}$ y su incertidumbre $u j$ se toman de la tabla III.

Cada valor aleatorio de $v_{i j}$ se introduce en la función $f$ de la ecuación (1) y se obtiene un valor aleatorio del mensurando $y_{i}=f\left(v_{i j}\right)$, donde $i=1,2,3, \ldots, 5000$. Una vez generados los 5000 valores aleatorios del mensurando se calcula la incertidumbre $u_{y}$ como desviación estándar para $n$ grados de libertad.

Ejecutando el algoritmo se tienen la media poblacional del mensurando $(y=\mu)$, la incertidumbre $u_{v}$ y la incertidumbre expandida $U$ al $95.45 \%$ de nivel de probabilidad con un factor de cobertura $k=2$.

$$
\begin{gathered}
y=1.4686610230 \\
u_{y}=0.2255427491 \\
U=0.4510854982
\end{gathered}
$$

La incertidumbre estándar relativa ISR en \% es:

$$
I S R=\frac{u_{y}}{y} \times 100=15.36 \%
$$

El contenido de aflatoxina B1 en la muestra de maní de exportación para un factor de cobertura al 95\% $(k=2)$ con el número de dígitos significativos es el siguiente:

$$
Y=(1.47 \pm 0.45) \mu g / K g(o n g / g)
$$

\section{Cálculo usando Excel 2007}

El principio de la simulación es el mismo que para el cálculo realizado en Maple. Se construye la matriz que se muestra en la tabla IV.

Tabla IV. Diseño matricial para el cálculo de la incertidumbre por SMC y resultados del cálculo.



En la fila Datos se introducen los valores experimentales de las variables $x_{j}$ y su incertidumbre $u_{j}$ respectiva (tabla III). En cada una de las columnas $z_{i j}$ se generaron las poblaciones de valores aleatorios normalizados $z$ (ver figura 7 en anexo).

Para generar estos valores se seleccionó Datos en la barra de herramientas de Excel 2007. Luego se seleccionaron sucesivamente las casillas Análisis de Datos y Generación de Números Aleatorios. Dentro de este procedimiento se completaron los datos siguientes:

a)Número de variables: 1

b)Cantidad de números aleatorios: 5000

c)Distribución: normal

d)Parámetros: media 0 desviación estándar 1

e)Iniciar con: 1

f)Opciones de salida-rango: se seleccionó la columna $z_{i j}$ a partir de $i=1$ hasta $n=5000$. 
Una vez generados los valores aleatorios de la columnas $z_{i j}$, se introdujo la fórmula $v_{i j}=x_{j}+z_{i j} u_{j}$ en cada columna etiquetada con $v_{i j}$, desde $\mathrm{i}=1$ hasta 5000 . En la columna $y_{i}$ se introduce la fórmula de la función $y_{i}=f\left(v_{i j}\right)$ de la ecuación (1) desde $i=1$ hasta 5000 para generar la población de los valores aleatorios del mensurando.

En las casillas correspondientes al mensurando $y$ se introduce la fórmula PROMEDIO (...) en el rango de la columna $y_{i}$ desde $i=1$ hasta 5000 . En la casilla correspondiente a la incertidumbre $u_{v}$, se introduce fórmula DESVEST(...) en el rango de la misma columna $y_{i}$, desde $i=1$ hasta 5000

Los resultados de la simulación numérica se muestran en las casillas correspondientes al mensurando $(y=1.47492964)$ y su incertidumbre $\left(u_{y}=0.22517952\right)$ de la tabla IV, representando la media y la incertidumbre estándar de la población. Los resultados se resumen a continuación:

$$
\begin{gathered}
I S R=15.27 \% \\
Y=(1.47 \pm 0.45) \mu g / K g \text { de aflatoxina B1 }
\end{gathered}
$$

Al comparar con los resultados del algoritmo ejecutado en Maple, se puede ver que no hay diferencias significativas. Es importante señalar que el programa de cálculo simbólico Maple tiene la ventaja de tener precisión ilimitada ${ }^{[18]}$.

\section{Comparación entre la técnica SMC y la ley de propagación de la incertidumbre}

En la tabla $V$ se reúnen los resultados del cálculo de la incertidumbre realizados por la simulación de Monte Carlo y los obtenidos por la ley de la propagación de la incertidumbre de la guía GUM ISO $1995^{[1]}$. Se puede observar claramente que las diferencias ocurren a partir del tercer decimal y dado que los dígitos significativos en el valor de incertidumbre están en los dos primeros, las diferencias no son significativas entre los 3 métodos.

No obstante, la simulación numérica tiene ventajas sobre el método analítico porque no solamente evita los cálculos de derivadas parciales, sino que puede evaluar la incertidumbre en modelos matemáticos difícilmente derivables ${ }^{[7-9,23]}$.

Tabla V. Resultados de las diferentes técnicas para el cálculo de la incertidumbre

\begin{tabular}{cccc}
\hline Técnica & $u_{y} \mu g / \mathrm{Kg}$ & ISR $\%$ & $\begin{array}{c}Y=(y \pm U) \mu g / K g \\
k=2 \text { al } 95.45 \%\end{array}$ \\
\hline SMC Maple & 0.225543 & 15.36 & $1.47 \pm 0.45$ \\
SMC Excel & 0.225180 & 15.27 & $1.47 \pm 0.45$ \\
GUM ISO 1995 & 0.224632 & 15.23 & $1.47 \pm 0.45$ \\
\hline
\end{tabular}

\section{Evaluación de la conformidad}

Ya se ha discutido que los valores máximos tolerados de la aflatoxina B1 varían según el país ${ }^{[1]}$, o del uso que se le vaya a dar al producto ${ }^{[17]}$. La conformidad del maní de exportación para consumo humano directo demandado por compradores de Inglaterra establece un valor límite de especificación superior (LES) $1.5 \mu \mathrm{g} / \mathrm{Kg}$. El reglamento europeo ${ }^{[25]}$ estipula que para alimentos de consumo humano directo el valor máximo (LES) de aflatoxina B1 permisible es de $2 \mu \mathrm{g} / \mathrm{Kg}$.

Para evaluar la conformidad se siguió el esquema del diagrama de flujo de la figura 6 , basado en el enfoque funcional de la evaluación de la conformidad establecido en la norma ISO $17000^{[14]}$.

Dependiendo de las especificaciones requeridas por los clientes, para el presente estudio, se dividen dos tipos 1) para LES $=1.5 \mu \mathrm{g} / \mathrm{Kg}$ y 2) para LES $=2 \mu \mathrm{g} / \mathrm{Kg}$. El método seleccionado acordado con el cliente es el de Cromatografía Líquida de Alto Desempeño con Detector de Fluorescencia (HPLC-FD). Este método presenta las siguientes características, tomadas de los datos de validación ${ }^{[26]}$, una linealidad de $99.96 \%$ en el rango de concentraciones de 0.7 a $5 \mu \mathrm{g} / \mathrm{kg}$ y un límite de detección (LD) de $0.1 \mu \mathrm{g} / \mathrm{Kg}$.

Basada en esta información y de acuerdo a los requisitos del cliente se verificó que el método es apto para el uso previsto y se procedió a la realización del ensayo para evaluar la incertidumbre utilizando la simulación de Monte Carlo. Los resultados arrojan que la incertidumbre estándar es $u_{y}=0.225 \mu \mathrm{g} / \mathrm{Kg}$ con un valor del mensurando o contenido de aflatoxina B1 en la muestra de $Y=(1.47 \pm 0.45) \mu \mathrm{g} / \mathrm{Kg}$. Se revisaron los resultados y se comparó con los requisitos de los compradores para finalmente confirmar el cumplimiento con la conformidad del producto de acuerdo a las especificaciones de los clientes.

El cumplimiento con las especificaciones se puede ver en la tabla VI. Según los resultados, caso 1, el maní cumple con los requisitos de las especificaciones del comprador que exige un contenido máximo de $2 \mu \mathrm{g} /$ $\mathrm{Kg}$ de aflatoxina B1; no obstante, el cumplimiento de la conformidad del producto para el cliente del caso 3 debe ser acordado con el cliente.

Tabla VI. Evaluación de la conformidad del maní para aflatoxina $B 1$.

\begin{tabular}{cccc}
\hline Caso & Resultados & Requisito (LES) & Cumplimiento \\
\hline 1 & $y+U=1.92<L E S$ & $\leq 2.0$ & SI \\
3 & $y=1.47=L E S$ & $\leq 1.5$ & SI, acordado \\
\hline
\end{tabular}




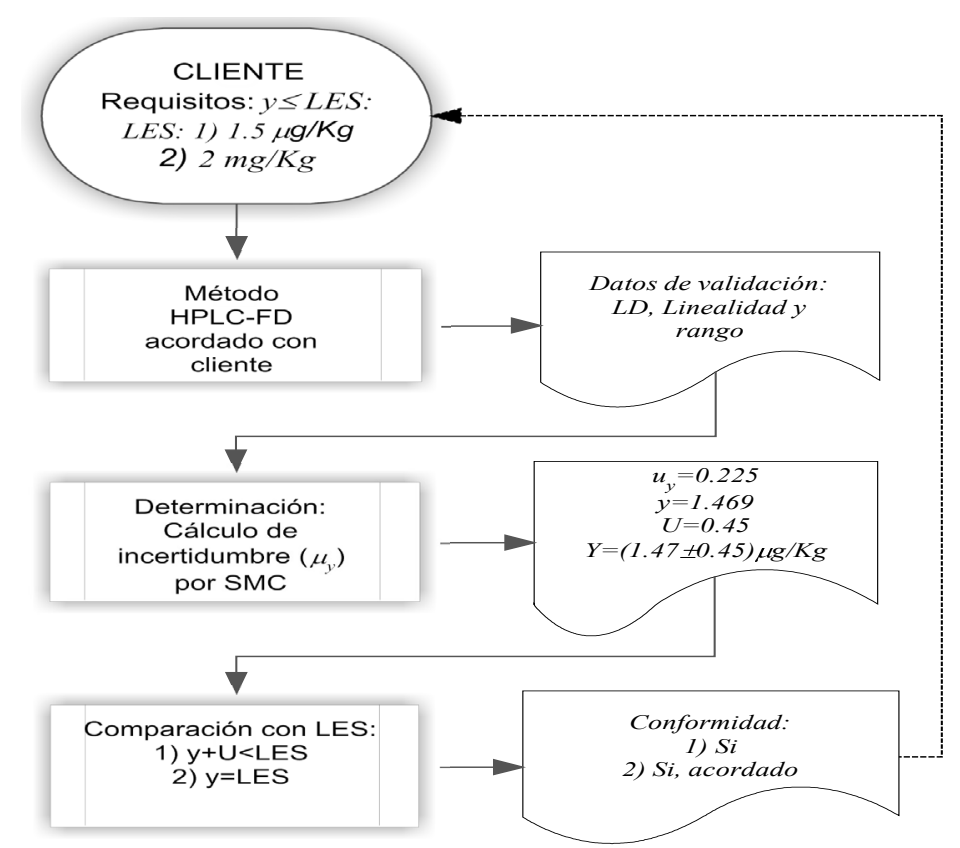

Figura 6: Enfoque funcional de la evaluación de la conformidad del maní de exportación.

En un artículo reciente ${ }^{[27]}$ se propone tomar en cuenta el límite de detección (LD) en los límites de especificación del producto. El cumplimiento de la conformidad se convierte en $Y \leq(L E S \pm L D)$. Para el presente estudio el límite de detección del método es $0.1 \mu \mathrm{g} / \mathrm{Kg}$.

De acuerdo a los criterios del cumplimiento de la especificación presentados en la tabla I, los requisitos son: $Y \leq(2.0-0.1)=1.9$ caso 2 y $Y \leq(1.5-0.1)=1.4$ caso 4 . En ambos casos se deduce que la conformidad del producto deberá ser acordado con el cliente. Si se reduce el nivel de probabilidad de la incertidumbre expandida, el nocumplimiento es posible. Esta propuesta no ha sido aún integrada en los criterios de la ILAC.

\section{CONCLUSIONES}

Se pudo demostrar que la simulación numérica de Monte Carlo es una técnica sencilla para evaluar la incertidumbre en la determinación del contenido de aflatoxina B1 por la técnica de HPLC-FD en una muestra de maní de exportación. El procedimiento general GUM ISO-EURACHEM fue modificado, sustituyendo la ley de propagación de la incertidumbre por la simulación numérica.

Se diseñó el algoritmo y se elaboró el programa en lenguaje Maple, generando valores aleatorios de las variables del modelo matemático. Luego se generó una población de la función que relaciona las variables y finalmente se calculó la media y la incertidumbre de la función como desviación estándar.
El contenido de aflatoxina B1 encontrado fue de $Y=(1.47 \pm 0.45) \mu \mathrm{g} / \mathrm{Kg}$ para un factor de cobertura de 2 al $95.45 \%$ de nivel de confianza. La simulación fue realizada también en hoja de cálculo Excel, obteniéndose los mismos resultados. Se demostró que esta técnica no difiere de la utilizada en el procedimiento GUM ISO 1995.

La incertidumbre expandida fue utilizada para la evaluación de la conformidad del maní que se exporta, siguiendo los criterios establecidos para el cumplimiento de las especificaciones del cliente de acuerdo a la guía de la Cooperación Internacional de Acreditación de Laboratorios (ILAC). Las especificaciones del producto cumple con los requisitos, $1.5 \mu \mathrm{g} / \mathrm{Kg}$ para compradores de Inglaterra y $2 \mu \mathrm{g} / \mathrm{Kg}$ para la comunidad europea.

Si se toma en cuenta el límite de detección en el límite superior de la especificación y se reduce el nivel de confianza en la incertidumbre expandida, el no cumplimiento es probable para ambos casos. Es importante destacar, que en el presente estudio no se tomó en cuenta la heterogeneidad de la muestra. En un mismo lote pueden existir grandes variaciones en el contenido de aflatoxinas.

\section{RECONOCIMIENTO}

Los autores agradecen al Laboratorio SER AGRO, S.A. por hacer posible la publicación del presente estudio, realizado dentro del contexto de la mejora continua que el laboratorio lleva a cabo para mantener la Acreditación 
que la Oficina Nacional de Acreditación (ONA- MIFIC) le ha otorgado desde el año 2001.

También agradecen al Dr. Ramiro Cáceres, profesor del Departamento de Matemáticas de la Facultad de Ciencias y Tecnología, por la revisión y sus valiosos aportes en los aspectos matemáticos del trabajo.

\section{REFERENCIAS BIBLIOGRAFICAS}

1. DELGADO G. y HERNÁNDEZ N., (2008), "Estimación de la incertidumbre en la determinación de aflatoxina B1 en maní de exportación por HPLC-FD", Universitas UNAN-León, en este mismo volumen.

2. BIPM, IEC, IFCC, IUPAC, OIML (1995). Guide for to the Expression of Uncertainty in Measurement (GUM), ISO, Ginebra.

3. NIST (1993). "Guidelines for evaluation and expressing the uncertainty of NIST measurement results", Gaithersburg, USA.

4. W.A. SCHMID y R.J. LAZOS. (2000), "Guía para estimar la incertidumbre de la medición", CENAM, México.

5. EURACHERM/CITAC GUIDE, (2000), Quantifying Uncertainty in Analytical Chemistry, 2da edición, UK.

6. DELGADO G y NAGEL B. (2008) "Un experimento sencillo para evaluar la incertidumbre siguiendo la guía GUM ISO 1995 y utilizando el cálculo simbólico MAPLE 11.0", Universitas UNAN-León, Vol 1, 2-1926.

7. BIPM, IEC, IFCC, IUPAC, ILAC, ISO, IUPAP y OIML, (2008). Suplement I to the Guide for to the Expression of Uncertainty in Measurement (GUM). Propagation of the distributions using a Monte Carlo Method, Paris.

8. MEIER P. y ZUND R., (2000), Statistical Methods in Analytical Chemistry, 2th Ed., John Wiley and Sons Inc., New York, pag. 163.

9. FERGUSON D., SIEPMANN I., THRULAR D., (1999), "Monte Carlo Methods in Chemical Physics", John Wiley and Sons, New York, pag. 13.

10. HERRADOR, M.A., GONZÁLEZ G.A., (2004),
"Evaluation of Measurement uncertainty in Analytical Assays by means of Monte Carlo Simulation", Talanta, Vol. 64, 6, 415-422.

11. OGREN P., DAVIS B y NICK G., (2001), "Curve fitting, confidence intervals and envelopes, correlations, and Monte Carlo visualization for multilinear problems in chemistry", J. C hem. Edu., Vol. 78, 6, 827-836.

12. ISO/IEC 17025(ES) (2005). "Requisitos Generales para la Competencia de los Laboratorios de Ensayo y de Calibración", Norma Internacional, Ginebra, Segunda Edición.

13. ILAC-G17-2002, (2003). "Introducción del concepto de incertidumbre de medición en el ensayo en asociación con la aplicación de la norma ISO/IEC 17025", Inter American Accreditation Cooperation (IAAC).

14. ISO/IEC 17000:2004 (ES), (2004). "Evaluación de la conformidad. Principios y vocabulario", Norma Internacional, Ginebra.

15. ISO/IEC 17025(ES) (2005). "Requisitos Generales para la Competencia de los Laboratorios de Ensayo y de Calibración", Norma Internacional, Ginebra, Segunda Edición.

16. ILAC-G8 (1996). "Guidelines on Assessment and Reporting of compliance with specification".

17. BHAT RV, VASATHI S., (1999), "Contaminación por Micotoxinas de Alimentos y Piensos", Tercera Conferencia Internacional FAO/OMS/PMA sobre Micotoxinas, Túnez. Disponible en http://www.fao. org/docrep/meeting/005/W5122S/w5122s0j.htm

18. CHAR B. W. (1992), Maple V, Springer Verlag, New York, pag. 132.

19. CHENEY W., KINCAID D., (1985), "Numerical Mathematics and Computing", Brooks/Cole Publishing Company, California, pag. 335.

20. GUTIERREZ H., SALAZAR R., (2004), "Control Estadístico de la Calidad y Seis Sigmas, McGrawHill Interamericana, México, pag. 150.

21. Asociación Latinoamericana de Integración (ALADI), "Acuerdo de superación a los obstáculos técnicos al comercio". Disponible en: www.puntofocal gov. 
ar/doc/acuerdo_marco_aladi.pdf. Acceso el 22 de diciembre del 2008.

22. SAOSA-01, (2007), Procedimiento para la determinación de Aflatoxinas por HPLC, Laboratorio SER-AGRO, Chinandega.

23. COWELLD.A., HOLKOMKE J.A., (1990), "Analytical applications of Monte Carlo", Anal. Chem., 62, 529A-542A.

24. SAOSA-PT-16, (2008), Procedimiento de evaluación de la incertidumbre, Laboratorio SER-AGRO, Chinandega.

25. DIRECTRIZ DEL PARLAMENTO EUROPEO, (2002), "Reglamentaciones del Parlamento Europeo". Disponible en internet y acceso el 1 de enero del 2009: http//www.knownmycotoxins.com/ es/regulations.htm.

26. SAOSA-PT-04, (2006), Reporte de validación del análisis de aflatoxinas por HPLC, Laboratorio SERAGRO, Chinandega.

27. DESIMONI, E. y BRUNETTI, B. (2006), "Considering uncertainty of measurement when assessing compliance or non-compliance with reference values given in compositional specifications and statutory limits: a proposal", Accred Qual Assur, 11, 363-366.

\section{ANEXO}

\section{Generación de una variable aleatoria por simulación} de Monte Carlo

Esta técnica consiste en generar $n(>1000$, generalmente 5000 ) variables aleatorias y se calcula la incertidumbre como desviación estándar ${ }^{[7,8]}$. La técnica se puede dividir en tres etapas: generación de un número aleatorio, transformación a un valor con distribución normal y generación de la variable aleatoria.

\section{Generación de un número aleatorio}

Los números aleatorios tienen una distribución uniforme, es decir rectangular, tal que $P(0 \leq R<1)=$ constante ${ }^{[7]}$. Los números aleatorios (o pseudoaletorios) pueden ser generados por diferentes técnicas[9]: congruencial lineal (LCG), Lagged-Fibonacci (ALFG), Shift-Register (SRGs), congruencial inversivo (ICG). El método LCG es el más utilizado por los compiladores $C$, lenguajes de programación como el FORTRAN ${ }^{[19]}$, cuyo algoritmo está basado en el siguiente principio: $x_{n}=a x_{n-1}-b(\bmod$ $N) ; N$ es entero muy grande $\left(\bmod 2^{j}\right), a$ y $b$ son enteros positivos seleccionados apropiadamente para obtener un buen generador; se inicia con un valor semilla $x_{0}$ que pertenece al conjunto $C=\{0,1,2, \ldots,(n-1)\}$; se construye la secuencia $y_{0}, y_{1}, y_{2}, \ldots$,donde $y_{i}=x / N$. De esta manera se generan números aleatorios $R$ en el intervalo $[0,1)$.

Las calculadoras científicas disponen de esta subrutina y fácilmente se pueden obtener números aleatorios. El cálculo se puede efectuar con Exce ${ }^{[20]}$, aplicando la función Aleatorio() o con la Distribución Uniforme en la casilla de Análisis de Datos. En Maple ${ }^{[18]}$ se utiliza la función rand().

\section{Transformación de un número aleatorio $\mathbf{R}$ en la desviación $z$ de la distribución normal}

Para convertir un número aleatorio $R$ de distribución uniforme en un valor normalizado $z$, se puede hacer a través de las probabilidades acumulativas $(C P)$ de la distribución normal[8]. El principio de la transformación se esquematiza en la figura 7 .

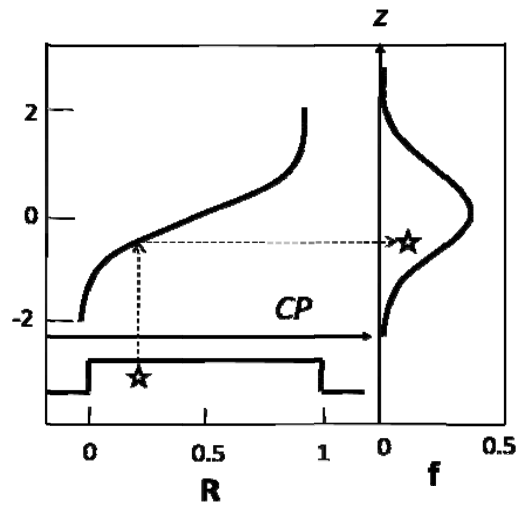

Figura 7. Para un número $R$ aleatorio con distribución rectangular corresponde un valor de probabilidad acumulada $C P$, la cual se transforma en la desviación $z$ de la distribución normal. $z$ es una función polinomial del logaritmo de $C P^{[8]}$.

La transformación de $R$ en $z$ se puede también realizar utilizando el algoritmo de Box-Muller ${ }^{[7,11]}$ generando 2 números aleatorios para cada valor de $z$ :

$$
\begin{aligned}
& z_{1}=\cos 2 \pi R_{2} \sqrt{-2 \ln R_{1}} \\
& z_{2}=\operatorname{sen} 2 \pi R_{2} \sqrt{-2 \ln R_{1}}
\end{aligned}
$$

El valor de $z$ a partir de R puede ser obtenido en Excel con Distribución Normal en la casilla de Análisis de Datos y en Maple con la subrutina stats [random,normald](). 

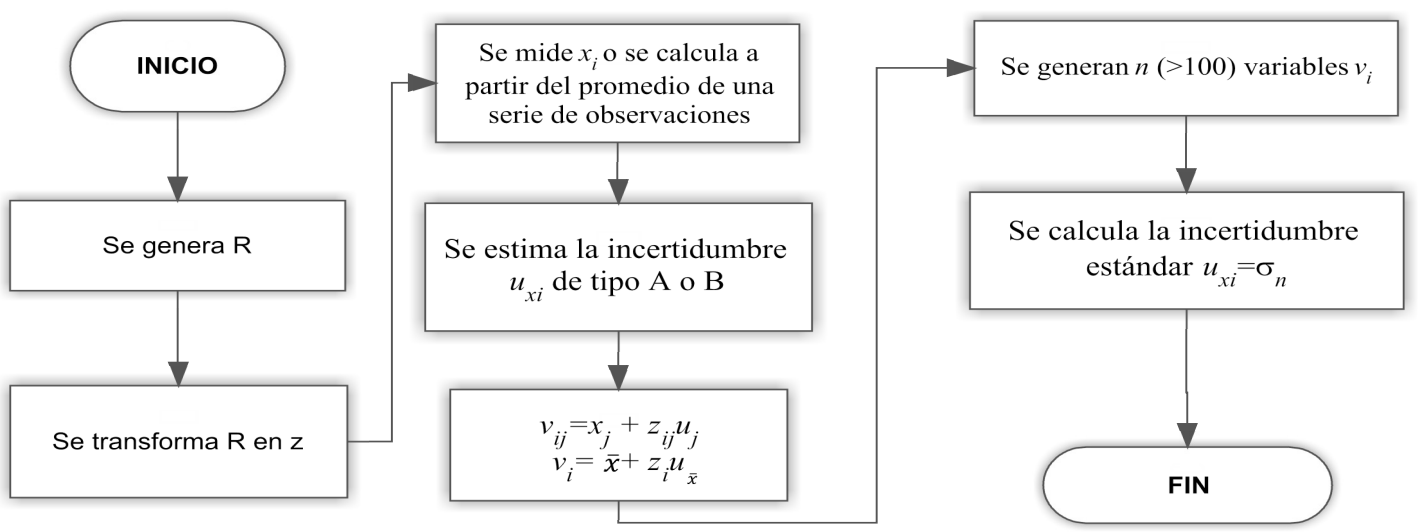

Figura 8. Algoritmo para generar una variable aleatoria y cálculo de la incertidumbre.

\section{Generación de la variable aleatoria y cálculo de la incertidumbre}

Una vez generado el valor $z$ a partir de $R$ se genera una variable aleatoria $v_{i}$. La operación se repite hasta generar $n$ variables aleatorias y se determina su incertidumbre. En el diagrama de flujo de la figura 8 se presenta el principio del algoritmo.

El cálculo de la variable $v_{i}$ se puede efectuar en Exce[ ${ }^{[20]}$. Se obtiene a partir de la Distribución Normal en la casilla Análisis de Datos, se le asigna el valor a la variable medida $x_{j}$ o el promedio $\bar{x}$ y su incertidumbre $u_{j}$. Luego se generan 5000 valores $v_{i}$ y se calcula la incertidumbre como desviación estándar para $n$ grados de libertad.

En Maple se pueden generar fácilmente 5000 variables aleatorias y evaluar la incertidumbre, utilizando el algoritmo de la figura 9 .

$$
\begin{aligned}
& S I=0 \\
& n=5000 \\
& \text { for } i \text { to } n \text { do } \\
& z[i]=\text { stats }[\text { random,normald }]() \\
& v[i]=x+z[i] u_{x} \\
& S 1=S 1+v[i] \\
& \text { od } \\
& S 2=0 \\
& \text { for } j \text { to } n \text { do } \\
& u=(S 2 / n)^{1 / 2}
\end{aligned}
$$

Figura 9. Algoritmo SMC en Maple para estimar la incertidumbre de una variable aleatoria. 